\title{
Modelagem do carbono e nitrogênio de um latossolo amarelo distrófico utilizando o Century
} 4.0

\section{Helton de Souza Silva',*, Adailson Pereira de Souza', Silvânia Maria de Sousa Gomes Nascimento², Mary Anne Barbosa de Carvalho ${ }^{1}$ e Léa Cristina de Medeiros ${ }^{1}$}

\author{
${ }^{1}$ Universidade Federal da Paraíba. Centro de Ciências Agrárias. Rodovia PB 079, km 12. \\ Bairro Universitário. Campus II. Areia-PB, Brasil (CEP 58397-000). *E-mail: \\ heltonssilva@gmail.com. \\ ${ }^{2}$ Universidade Federal da Paraíba. Centro de Ciências Humanas Sociais e Agrárias. Campus \\ III. Bananeiras-PB, Brasil (CEP 58220-000).
}

Resumo. A remoção da vegetação nativa e introdução de práticas agrícolas aumenta a decomposição da matéria orgânica do solo, adicionalmente, ocorre redução da produção primária pela retirada das florestas, culminando em elevadas perdas nos estoques de carbono $(\mathrm{C})$ e nitrogênio $(\mathrm{N})$ do solo. Objetivou-se com o presente trabalho mensurar o impacto das mudanças no uso do solo nos estoques de $\mathrm{C}$ e $\mathrm{N}$ e avaliar a eficiência do modelo Century 4.0 em simular os estoques de carbono e nitrogênio para as condições da Microrregião do Brejo Paraibano. Para realizar as simulações utilizou-se os dados de um experimento de longa duração realizado na Estação Experimental Chã-de-Jardim, do Centro de Ciências Agrárias (CCA/UFPB). A parametrização do modelo foi realizada com os dados de solo, clima e ajuste na entrada de N. Inicialmente, realizou-se a simulação de equilíbrio por um período de 6.000 anos para Floresta Tropical Úmida Montana, para obtenção de estoques estáveis de C e N. Após o equilíbrio das variáveis de saída, realizou-se as simulações do diferentes sistemas de uso do solo e dos tratamentos com as

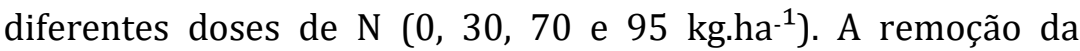
floresta e implantação da agricultura causa redução do estoque de carbono e nitrogênio do solo, sendo o compartimento lento o que tem maior perda absoluta. A adubação nitrogenada na cultura do milho em plantio direto na dose de $95 \mathrm{~kg} \mathrm{ha}^{-1}$ favorece a manutenção do estoque de carbono do solo. O Century é adequado para simulação do estoque de carbono orgânico do solo na Microregião do Brejo Paraibano. Já para o estoque de nitrogênio o modelo não é indicado.

Palavras-chave: Modelagem ambiental; Matéria orgânica do solo; Adubação nitrogenada.

Abstract. Carbon and nitrogen modelling of an oxisol using Century 4.0. The removal of native vegetation and the
Recebido

$11 / 12 / 2020$

Aceito

30/05/2021

Disponível on line $31 / 05 / 2021$

Publicado

$31 / 08 / 2021$

Acesso aberto

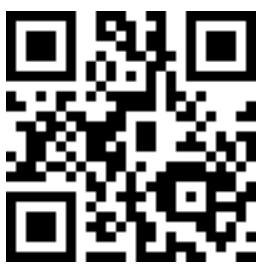

ORCID

D 0000-0003-0021-5610 Helton de Souza Silva

D 0000-0003-2049-6069 Adailson Pereira de Souza

(D) 0000-0001-8574-5383

Silvânia Maria de

Sousa Gomes

Nascimento

ISSN 2359-1412/RBGAS-2020-0176/2021/8/19/4/711

Rev. Bras. Gest. Amb. Sustent.

http://revista.ecogestaobrasil.net 
introduction of agricultural practices increases the decomposition of soil organic matter, in addition, there is a reduction in primary production by clearing forests, resulting in high losses in soil carbon (C) and nitrogen (N) stocks. The objective of this study was to measure the impact of land use changes on $\mathrm{C}$ and $\mathrm{N}$ stocks and to evaluate the efficiency of the Century 4.0 model in simulating carbon and nitrogen stocks for Microrregião do Brejo Paraibano conditions. To perform the simulations, we used data from a long-term experiment conducted at the Chã-do-Jardim Experimental Station of the Centro de Ciências Agrárias (CCA/UFPB). Parameterization of the model was carried out using soil, climate and $\mathrm{N}$-input data. Initially, a 6000-year equilibrium simulation was carried out for Montana Rainforest to obtain stable $\mathrm{C}$ and $\mathrm{N}$ stocks. After balancing the output variables, simulations of different land use systems and treatments with different $\mathrm{N}$ rates $\left(0,30,70\right.$ and $\left.95 \mathrm{~kg} \cdot \mathrm{ha}^{-1}\right)$ were performed. Removal of the forest and implementation of agriculture causes a reduction in soil carbon and nitrogen stock, with the slow compartment having the highest absolute loss. Nitrogen fertilization in corn at a dose of $95 \mathrm{~kg}^{-h^{-1}}$ promotes on maintenance of soil carbon and nitrogen stock. Century proved to be suitable for simulating soil organic carbon stock. For nitrogen stock, the model is not suitable.

Keywords: Environmental modelling; Soil organic matter; Nitrogen fertilization.
D) $0000-0003-0563-6190$ Mary Anne Barbosa de Carvalho

(1) 0000-0003-2100-4564

Léa Cristina de

Medeiros

\section{Introdução}

O solo é um dos maiores reservatórios de carbono orgânico do planeta, chegando a reter aproximadamente $1.415,7 \mathrm{Pg}$, a vegetação por sua vez é responsável por reter 496,6 Pg (Scharlemann et al., 2014) e a atmosfera contribui com 839 Pg de carbono (Kayler et al., 2019). Essas três fontes interagem simultaneamente e a vegetação e o solo funcionam como dreno e/ou fonte do carbono atmosférico, que por sua vez é indispensável para a produção primária. Portanto, as práticas de uso do solo são fundamentais para a manutenção do equilíbrio entre o sistema solo-planta-atmosfera.

Práticas de uso do solo que causam perturbação do equilíbrio, como a remoção da vegetação nativa e introdução da agricultura, aumentam a emissão líquida de $\mathrm{CO}_{2}$, causando declínio no estoque de carbono orgânico do solo. Adicionalmente, ocorre a redução da produção primária pela retirada das florestas, causando à quebra do equilíbrio dinâmico e um novo equilíbrio é atingido num nível que varia de acordo com as novas condições do sistema de manejo adotado (Ontl e Schult, 2012).

A utilização de métodos conservacionistas de preparo do solo, juntamente com sistemas de culturas, que adicionam elevada quantidade de material, propicia, respectivamente, menores taxas de perdas e maiores taxas de adição de matéria orgânica ao solo (Costa et al., 2008), sendo uma das alternativas para recuperar ou manter o teor de matéria orgânica do solo.

Outro elemento determinante na dinâmica da matéria orgânica do solo é o Nitrogênio. Os ciclos do Carbono e do Nitrogênio estão intimamente relacionados. Qualquer redução no estoque de carbono orgânico do solo, causado pelas alterações no seu uso, reflete diretamente no estoque de nitrogênio, o que leva a um declínio 
proporcional ao do carbono (Bortolon et al., 2009), pelo consequente aumento líquido na emissão de $\mathrm{N}_{2} \mathrm{O}$ (Bayer et al., 2015).

Os efeitos das práticas de uso sobre a dinâmica do carbono e nitrogênio do solo podem ser preditos com a utilização de modelos computacionais como o Century, permitindo que possíveis efeitos negativos sejam antecipados. Apesar do Century ter sido criado para ambientes de clima temperado, pode ser utilizado com segurança em regiões de clima tropical (Oliveira et, 2014; Althoff et al., 2018).

Portanto, objetivou-se com a presente pesquisa mensurar o impacto das mudanças no uso do solo nos estoques de $\mathrm{C}$ e $\mathrm{N}$ de um Latossolo Amarelo distrófico, bem como avaliar a eficiência do modelo Century 4.0 em simular os estoques de carbono e nitrogênio para as condições da Microrregião do Brejo Paraibano.

\section{Material e métodos}

O experimento utilizado para realizar as simulações dos estoques de carbono e de nitrogênio do solo foi realizado na Estação Experimental Chã-de-Jardim, pertencente ao Centro de Ciências Agrárias, da Universidade Federal da Paraíba (CCA/UFPB). Situada no Município de Areia, Estado da Paraíba, Nordeste do Brasil, nas seguintes coordenadas geográficas de referência $6^{\circ} 58^{\prime} 0,9^{\prime \prime} \mathrm{S}$ e $35^{\circ} 43^{\prime} 55^{\prime \prime} \mathrm{O}$, com altitude média de $620 \mathrm{~m}$.

0 clima na região de estudo é do tipo Tropical Chuvoso, com verão seco. A estação chuvosa se inicia em janeiro/fevereiro com término em setembro, podendo se adiantar até outubro, com precipitação média anual de 1.358,4 mm (AESA, 2018).

Como referência para o estoque de carbono e nitrogênio do solo e para realizar a simulação de equilíbrio, utilizou-se uma área com floresta tropical de altitude, a cerca de $300 \mathrm{~m}$ do experimento.

Tabela 1. Descrição do manejo adotado na área experimental localizada na estação experimental Chã-do-Jardim, Centro de Ciências Agrárias, Universidade Federal da Paraíba.

\begin{tabular}{|c|l|c|}
\hline Períodos & \multicolumn{1}{|c|}{ Histórico da área } & Ano \\
\hline 1 & $\begin{array}{l}\text { O bloco um foi constituído de mata nativa (floresta tropical úmida de } \\
\text { altitude), utilizado para realizar a simulação de equilíbrio. A qual foi } \\
\text { desmatada em 1950. }\end{array}$ & Até 1950 \\
\hline 2 & $\begin{array}{l}\text { A partir de 1951 foi implantada agricultura em plantio convencional, } \\
\text { sendo cultivado com as principais culturas da região (Milho, Feijão, } \\
\text { Mandioca e Cana-de-açúcar). }\end{array}$ & $1951-1985$ \\
\hline 3 & $\begin{array}{l}\text { A partir de 1986 foi implantado um cultivo de amoreira, } \\
\text { permanecendo na área até 1995. }\end{array}$ & $1986-1995$ \\
\hline 4 & $\begin{array}{l}\text { A partir de 1996 a área foi utilizada para cultivo de milho e feijão em } \\
\text { sistema de cultivo convencional, com o plantio sendo realizado entre } \\
\text { março e abril e a colheita entre julho e agosto. E durante o período de } \\
\text { setembro a março a área permanecia em pousio, com o solo coberto } \\
\text { por vegetação espontânea. }\end{array}$ & $1996-2005$ \\
\hline 5 & $\begin{array}{l}\text { De 2006 a 2012 a área permaneceu em pousio, com o solo coberto } \\
\text { por vegetação espontânea. }\end{array}$ & $2006-2012$ \\
\hline 6 & $\begin{array}{l}\text { A partir de 2013 foi instalado um experimento com adubação } \\
\text { nitrogenada na cultura do milho, cultivado em sistema de plantio } \\
\text { direto na palha. Com o plantio sendo realizado sempre entre março e } \\
\text { abril e a colheita realizada entre julho e agosto. Durante o período de } \\
\text { setembro a março a área permanecia em pousio. }\end{array}$ & $2013-2067$ \\
\hline
\end{tabular}

Rev. Bras. Gest. Amb. Sustent., 2021, vol. 8, n. 19, p. 711-723. 
Com o objetivo de conhecer o histórico de manejo da área de estudo, foram realizadas entrevistas com servidores da universidade que trabalhavam na estação experimental, sendo descrito cinco períodos distintos de manejo, adotados na área antes do início do experimento (Tabela 1).

O experimento com adubação nitrogenada foi implantando em 2013, sendo realizado a cada ano de cultivo a aplicação de três doses de nitrogênio $(\mathrm{N})$ na forma de

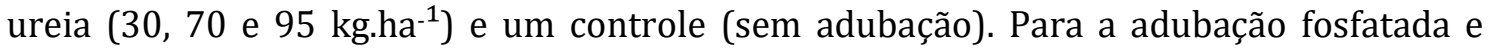
potássica foram utilizadas as mesmas doses para todos os tratamentos, 80 kg.ha-1 de $\mathrm{P}_{2} \mathrm{O}_{5}$ na forma de superfosfato simples e $56 \mathrm{~kg} \mathrm{ha}^{-1}$ de $\mathrm{K}_{2} \mathrm{O}$ na forma de $\mathrm{KCl}$, respectivamente. A variedade de milho AG 1051 foi semeada no espaçamento de $1,0 \times 0,2 \mathrm{~m}$ perfazendo um stand de 50.000 mil plantas por hectare.

Cada unidade experimental foi composta por sete linhas de plantio com dez metros de comprimento, sendo considerado como área útil $40 \mathrm{~m}^{2}$ da parte central de cada parcela. As parcelas foram dispostas em delineamento em blocos ao acaso, com cinco repetições. 0 solo da área experimental é classificado como Latossolo Amarelo distrófico (Santos et al., 2018).

O plantio foi realizado sobre a palhada, 30 dias após a aplicação do glifosato nas plantas infestantes, para isso foram abertos sulcos com $10 \mathrm{~cm}$ de profundidade, onde foi feita a adubação de fundação e posteriormente semeado o milho, tendo-se o cuidado de cobrir o adubo com terra. Aos 40 dias após o plantio foi realizado o controle mecânico das plantas infestantes, ao término foi realizada a adubação de cobertura.

A amostragem do solo foi feita ao final de cada ciclo do milho para realizar as análises químicas e físicas, para isso coletou-se três amostras simples por unidade experimental na profundidade de $0-20 \mathrm{~cm}$, com auxílio de trado holandês. $\mathrm{Na}$ área de mata nativa procedeu-se a subdivisão em cinco quadrantes de $100 \mathrm{~m}^{2}$ cada, em cada quadrante realizou-se a coleta de 10 amostras simples, na profundidade de $0-20 \mathrm{~cm}$.

A densidade do solo foi determinada utilizando-se amostras indeformadas pelo método do cilindro, o teor de carbono orgânico foi determinado por oxidação com dicromato, o pH foi aferido em água na proporção 1:2,5 e a textura foi obtida pelo método do densímetro (Teixeira et al., 2017). 1995).

O nitrogênio total do solo foi determinado pelo método de Kjeldahl (Tedesco et al.,

Tabela 2. Parâmetros de entrada obtidos na estação meteorológica localizada no Centro de Ciências Agrárias da Universidade Federal da Paraíba.

\begin{tabular}{|l|c|c|c|}
\hline Mês & Prec $^{\mathbf{1}}(\mathbf{m m})$ & TMax $^{\mathbf{1}}\left({ }^{\circ} \mathbf{C}\right)$ & TMin $^{\mathbf{1}}\left({ }^{\circ} \mathbf{C}\right)$ \\
\hline Janeiro & 80,06 & 28,80 & 20,02 \\
\hline Fevereiro & 99,33 & 28,65 & 20,26 \\
\hline Março & 140,67 & 28,41 & 20,54 \\
\hline Abril & 162,43 & 27,64 & 20,64 \\
\hline Maio & 170,89 & 26,39 & 20,03 \\
\hline Junho & 208,02 & 24,64 & 19,00 \\
\hline Julho & 203,22 & 24,02 & 18,14 \\
\hline Agosto & 130,01 & 24,61 & 17,78 \\
\hline Setembro & 59,27 & 25,98 & 18,32 \\
\hline Outubro & 24,33 & 27,73 & 19,01 \\
\hline Novembro & 28,33 & 28,68 & 19,43 \\
\hline Dezembro & 42,13 & 29,15 & 19,83 \\
\hline
\end{tabular}

${ }^{1}$ Média histórica de 1987 a 2017. Prec = Precipitação; TMax = temperatura máxima; TMin = temperatura mínima. 
As variáveis específicas do local (sítio) utilizadas foram o $\mathrm{pH}(4,2)$, granulometria (560, 30 e 410 g.kg $^{-1}$ de areia, silte e argila, respectivamente) e densidade do solo $\left(1,26 \mathrm{~kg} \cdot \mathrm{dm}^{-3}\right)$ de referência. As informações climáticas utilizadas foram a média histórica de 1987 até 2017, obtidas na estação meteorológica localizada no Centro de Ciências Agrárias da Universidade Federal da Paraíba (CCA/UFPB) (Tabela 2).

Os parâmetros de entrada de $\mathrm{N}$ no sítio foram definidos pelos valores descritos por Reed et al. (2011) e Dentener et al. (2006).

Para representar melhor o bioma em estudo foi realizado a calibração nos parâmetros fixos (FIX.100), sendo feita a parametrização para biomas de clima tropical úmido que tenham relação C/N do solo > 15, conforme descrito por Parton et al. (2001).

A calibração para a mata nativa (TREE.100) foi realizada utilizando os parâmetros do default e do Century Parameterization Workbook (Parton et al., 2001), para florestas tropicais.

Após o início do cultivo do milho em sistema de plantio direto, para realizar a calibração da produção de biomassa do milho, considerou-se a produção média de três anos, período no qual não ocorreram limitações hídricas. A produção média de grãos foi, respectivamente, de 5.055, 9.093, 11.070 e 13.295 kg.ha-1 para as doses de adubação nitrogenada de $0,30,70$ e $95 \mathrm{~kg}^{-h^{-1}}$. Considerou-se o teor médio de carbono da biomassa do milho igual 55\%, obtido pela determinação do C pelo método de combustão via seca. Os valores de biomassa produzida acima do solo (PRDX) de 92,67, 166,70, 202,95 e 243,74 g de $\mathrm{C} / \mathrm{mês} / \mathrm{m}^{2}$ para as doses de nitrogênio de 0,30, 70 e 95 kg.ha-1, respectivamente.

Os demais parâmetros de entrada utilizados para a calibração da cultura do milho foram propostos por Parton et al. (2001).

Nos diferentes cenários de adubação realizou-se a calibração da entrada de fertilizantes (FERT.100), de acordo com as doses de N e Superfosfato Simples.

O PRDX durante o pousio de cada ano agrícola foi determina pela amostragem de $1 \mathrm{~m}^{2}$ em três pontos distintos, sendo considerada a produção de massa seca, a média das três repetições, a qual foi de $12.500 \mathrm{~kg} \cdot \mathrm{ha}^{-1}$, sendo considerado o teor de carbono igual a $55 \%$. Os demais parâmetros utilizados na calibração foram originados do default (Parton et al., 2001).

As simulações dos diferentes sistemas de uso do solo foram realizadas tendo como ponto de partida a simulação de equilíbrio de 6000 anos, sendo simuladas as condições de desmatamento da área, introdução da agricultura em sistema convencional (PRDX=250), plantio de amoreira $(P R D X=100)$, pousio $(P R D X=300)$ e os diferentes cenários de adubação nitrogenada.

Para averiguar a adequação do modelo em simular os estoques de carbono e nitrogênio orgânico do solo realizou-se o procedimento estatístico denominado LOFIT (Smith et al., 1997).

\section{Resultados e discussão}

Os compartimentos de carbono do solo atingiram o equilíbrio após 3.000 anos da simulação. 0 compartimento lento foi o que teve maior contribuição para o estoque total de carbono do solo, seguido pelo compartimento passivo e ativo, com uma contribuição de 54; 44 e 2\%, respectivamente (Figura 1A). Para os compartimentos de nitrogênio o equilíbrio foi alcançado após 4.500 anos da simulação, sendo o compartimento passivo o de maior contribuição, seguido do lento e ativo (Figura 1B).

Rev. Bras. Gest. Amb. Sustent., 2021, vol. 8, n. 19, p. 711-723. 

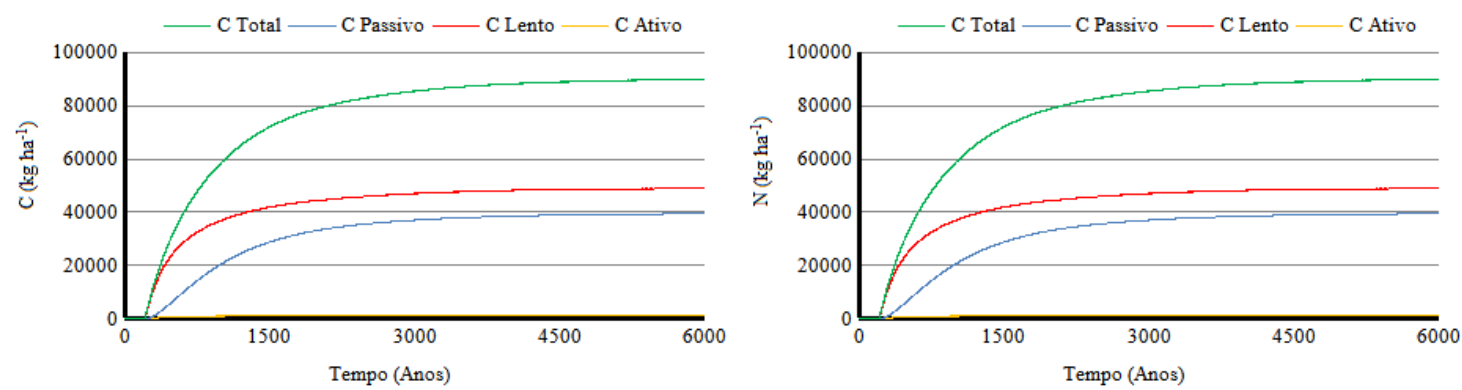

Figura 1. Simulação de equilíbrio do estoque de C (A) e N (B) em Latossolo Amarelo distrófico na camada de 0-20 cm com Floresta Tropical Úmida Montana.

A maior contribuição do estoque de $\mathrm{C}$ pelo compartimento lento é devido a elevada produção de biomassa, típica de florestas tropicais, associado ao pH ácido $(4,2)$, condição limitante para o processo de estabilização do carbono do compartimento lento (Zech et al., 1997). Esses resultados corroboram com os reportados por Miranda et al. (2007) e Barreto et al. (2008), os quais relataram que em área com Mata Atlântica há maior proporção da fração lenta do carbono orgânico do solo.

0 estoque de carbono simulado da mata nativa foi semelhante a média dos valores medidos (Figura 2A). Para o estoque de nitrogênio houve superestimação dos valores simulados, sendo 13\% maior que o estoque de nitrogênio medido (Figura 2B).
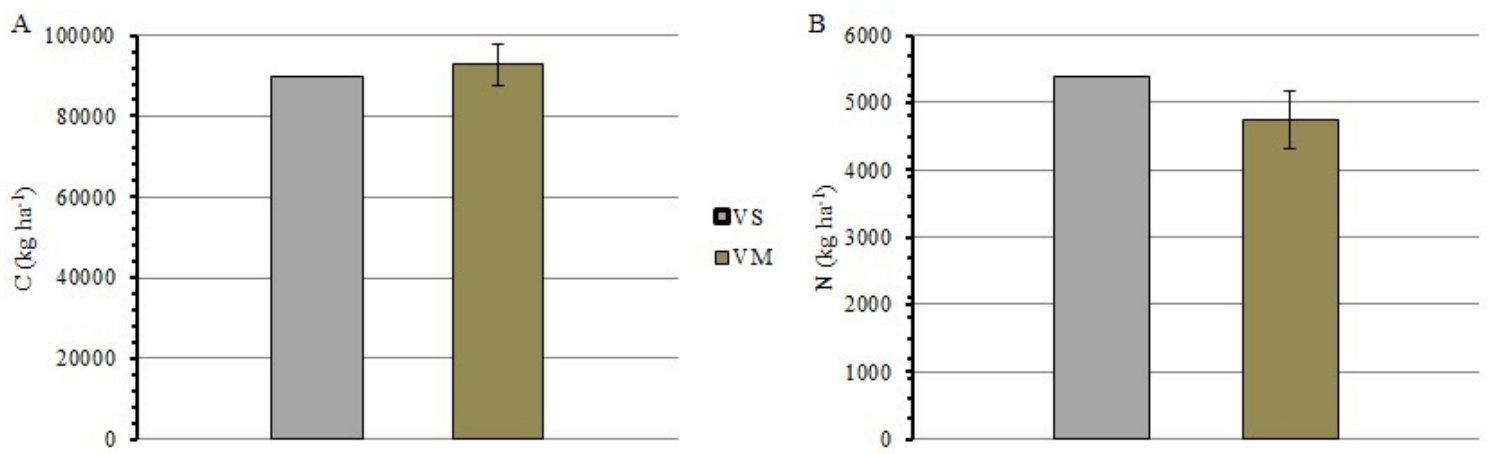

Figura 2. Valores medidos (VM) e simulados (VS) do estoque de C (A) e N (B) dum Latossolo Amarelo distrófico na camada de $0-20 \mathrm{~cm}$ com Floresta Tropical Úmida Montana.

Com o desmatamento da floresta e inserção da agricultura, houve redução do estoque de carbono do solo. Do ano de 1950 (Desmatamento) até o ano anterior ao início do experimento com adubação (2012) o decréscimo total do estoque de carbono orgânico do solo foi de 52\% (Figura 3). A fração lenta do carbono orgânico do solo reduziu 85\% após o desmatamento até o ano de 2012 , o que corresponde à $46 \%$ do estoque de carbono orgânico. Neste mesmo período a fração passiva declinou apenas 5\% (Figura 3). 

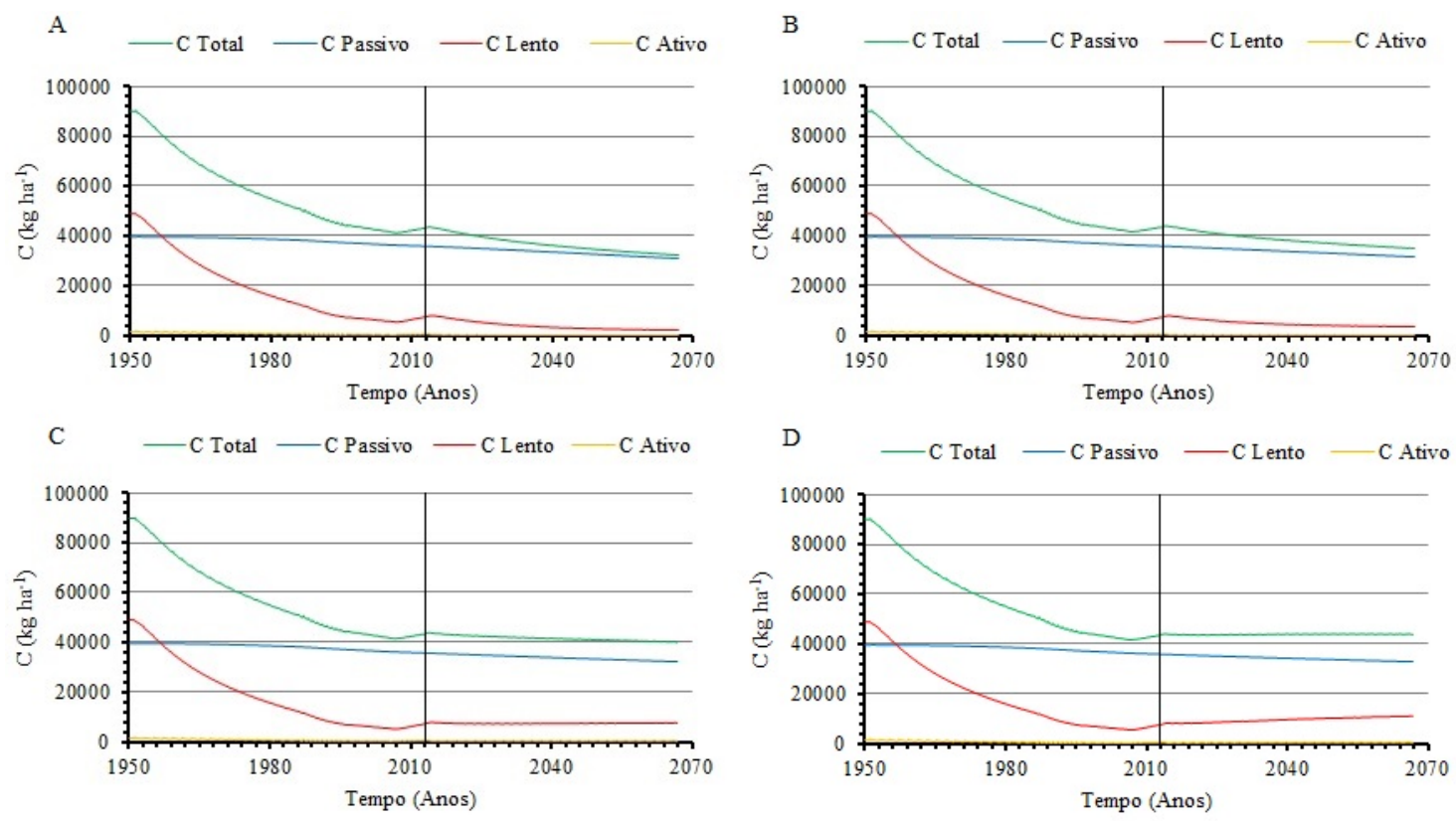

Figura 3. Simulação do estoque de $C$ dum Latossolo Amarelo distrófico na camada de 0-20 cm após o desmatamento e submetido a doses de nitrogênio, 0 (A), 30 (B), 70 (C) e 95 kg.ha-1 (D)

Considerando que o compartimento lento é mais lábil que o compartimento passivo, sendo o lento representado pela matéria orgânica leve, com o tempo de reciclagem estimado entre 20 e 40 anos e o passivo é representado pelas substâncias húmicas em avançado grau de estabilização química, protegido fisicamente por minerais de argila ou oclusas em criptoporos ou microporos, as quais podem alcançar tempos de reciclagem entre 200 a 500 anos (Tornquist et al., 2009). Portanto, o compartimento de $C$ lento é mais sensível às alterações de manejo do solo.

No experimento de adubação nitrogenada, iniciado a partir do ano de 2013, constatou-se que apenas o tratamento com aplicação de $95 \mathrm{~kg} \cdot \mathrm{ha}^{-1}$ de $\mathrm{N}$ contribuiu para a manutenção do carbono do solo, pois promoveu acréscimo no compartimento lento (Figura 3D).

0 estoque de nitrogênio do solo também reduziu após o desmatamento, semelhante ao que ocorreu ao estoque de carbono (Figura 4). A partir do ano de 2013 foi possível observar que apenas o tratamento com aplicação de $95 \mathrm{~kg}^{-h^{-1}}{ }^{-1} \mathrm{~N}$, proporcionou incremento no estoque de nitrogênio do compartimento lento, favorecendo para que houvesse manutenção do estoque total de nitrogênio do solo (Figura 4D).

A manutenção do estoque de carbono e nitrogênio do solo constatada com a plicação da dose de $95 \mathrm{~kg} \mathrm{ha}^{-1} \mathrm{~N}$ ocorreu devido a maior produção de biomassa do milho proporcionada pela maior disponibilidade de $\mathrm{N}$, somado ao efeito do sistema conservacionista utilizado a partir do ano de 2013, no entanto, não foi eficiente em recuperar o estoque de carbono existente antes do desmatamento. Leite; Mendonça e Machado (2004) reportaram que após o desmatamento e inserção de cultivo convencional há declínio nos estoques de carbono e nitrogênio orgânico do solo, sendo possível a recuperação desse estoque com adubação orgânica. 

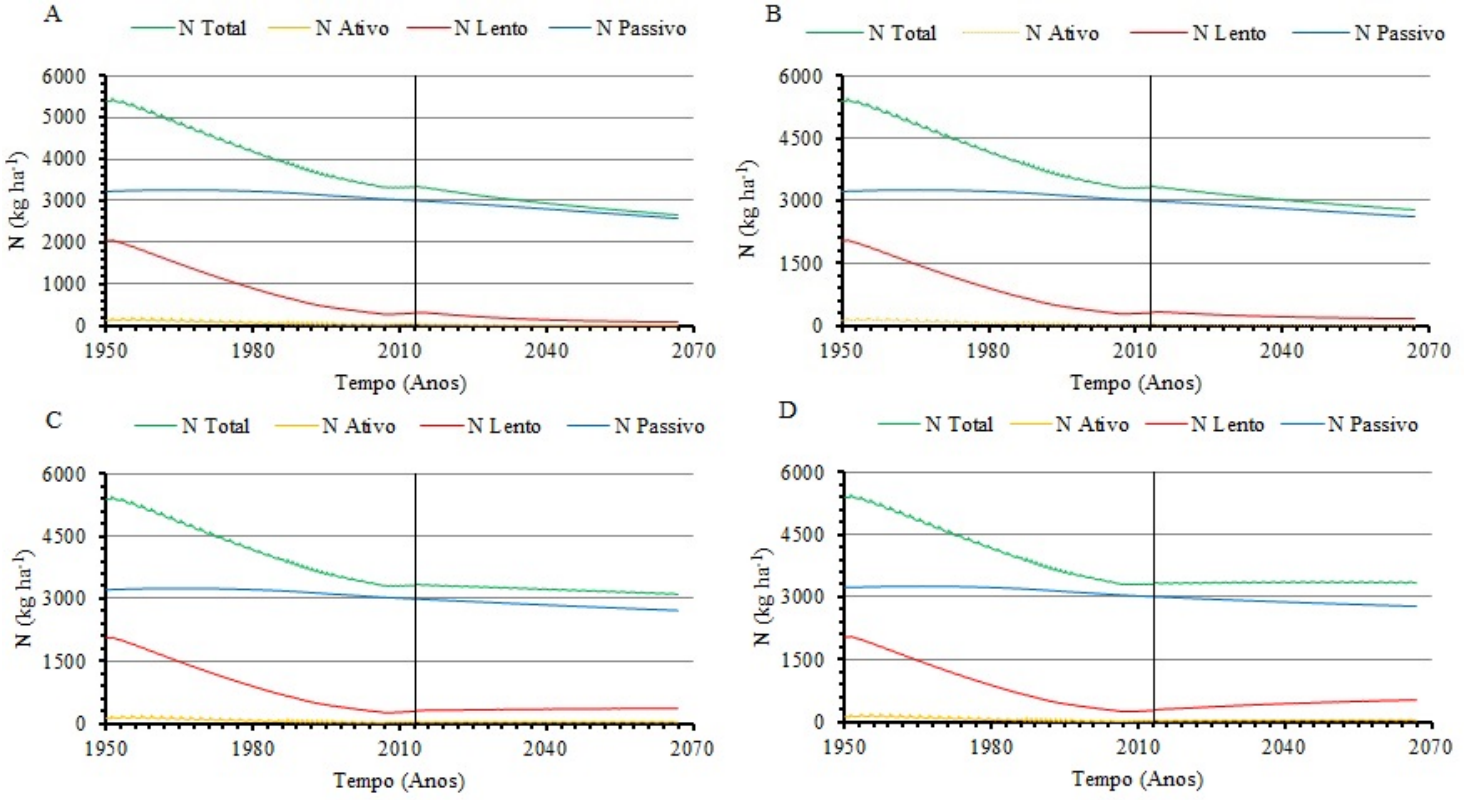

Figura 4. Simulação do estoque de N dum Latossolo Amarelo distrófico na camada de 0-20 cm após o desmatamento e submetido a doses de nitrogênio, 0 (A), 30 (B), 70 (C) e 95 kg.ha-1 (D).

0 estoque de carbono orgânico do solo simulado foi semelhante aos valores medidos. Nos anos de 2013 e 2014 a diferença entre o estoque de carbono simulado e medido foi inferior à 10\% (Figura 5 A e B). Nos anos de 2016 e 2017 houve maior diferença entres os valores simulados e medidos do estoque de carbono orgânico do solo (Figura $5 \mathrm{C}$ e D). Apesar de haver diferença entre os valores simulados e medidos do carbono orgânico do solo em torno de $10 \%$, os valores simulados são considerados aceitáveis, devido a adequação do modelo constatada através do procedimento LOFIT.

Os valores simulados de nitrogênio do solo foram subestimados pelo modelo, em média 25\%, em todos os tratamentos tanto no ano de 2016 como no ano de 2017 (Figura 6 A e B). Esse problema deve ter ocorrido devido a alteração na entrada de nitrogênio, através da fixação biológica e deposição atmosférica, ocasionada pelas mudanças de uso do solo, após o desmatamento. Uma vez que essa entrada de nitrogênio não pode ser alterada para cada sistema de manejo, por ser um dos parâmetros de entrada do sítio.

Através do teste de $\mathrm{F}(\mathrm{P} \leq 0,05)$ foi possível constatar que não houve diferença significativa entre os valores medidos e simulados do estoque de carbono do solo (Tabela 1). A diferença total entre os valores medidos e simulados foi calculada pela raiz do quadrado médio do erro (RMSE), e sua significância testada num intervalo de $95 \%$ de confiança $\left(\operatorname{RMSE}_{(95 \%)}\right)$, sendo constatado que apenas os valores simulados do estoque de carbono do solo de referência estão dentro do intervalo dos valores medidos, com $95 \%$ de confiança (Tabela 3). 

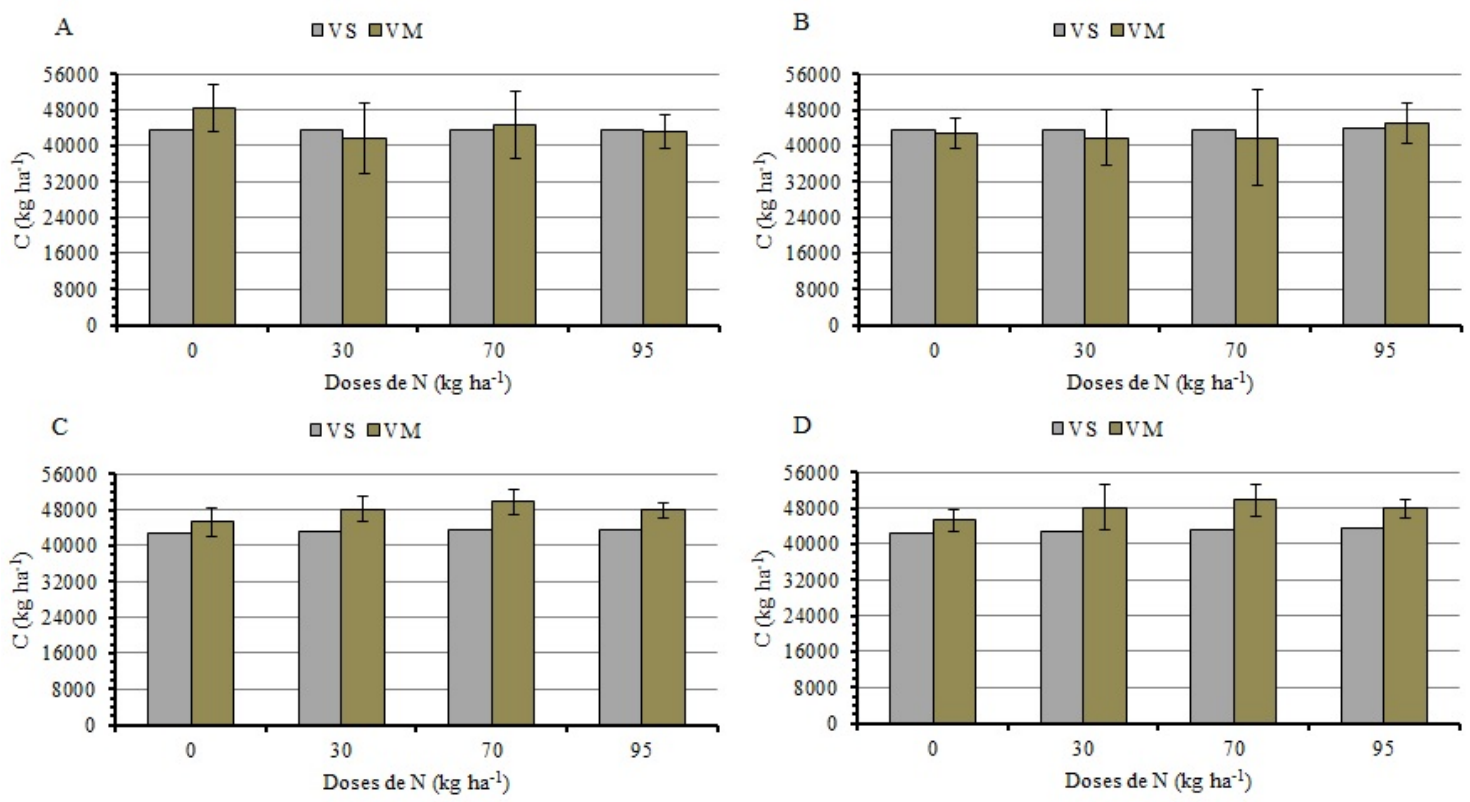

Figura 5. Valores medidos (VM) e simulados (VS) do estoque de C dum Latossolo Amarelo distrófico na camada de 0-20 cm submetido a doses de nitrogênio (0, 30, 70 e $\left.95 \mathrm{~kg} \mathrm{ha}^{-1}\right)$ nos anos de 2013 (A), 2014 (B), 2016 (C) e 2017 (D).
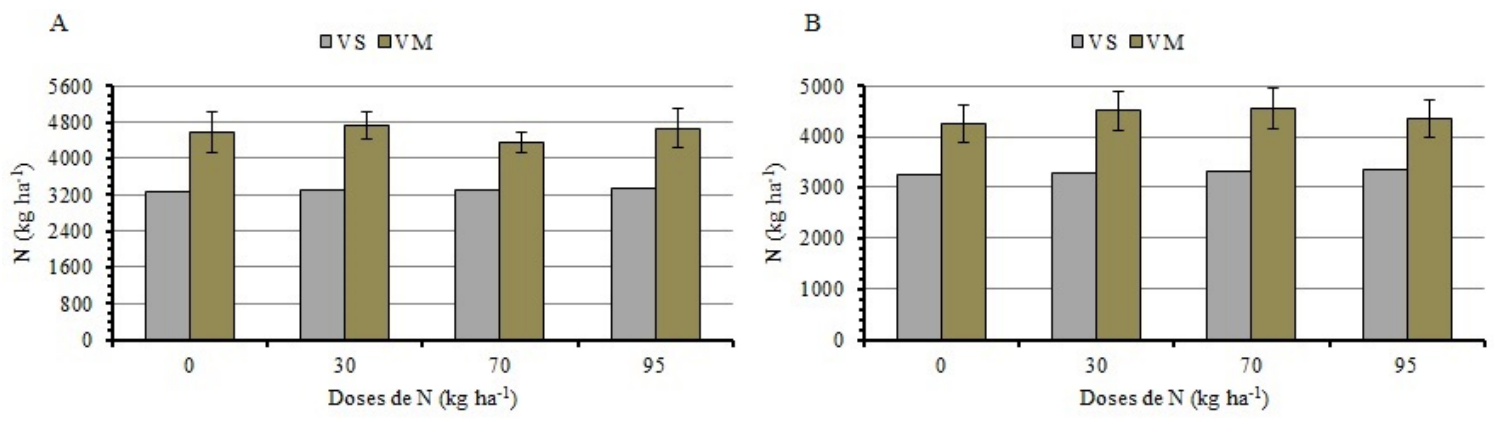

Figura 6. Valores medidos (VM) e simulados (VS) do estoque de $\mathrm{N}$ dum Latossolo Amarelo distrófico na camada de 0-20 cm submetido a doses de nitrogênio (0, 30, 70 e $95 \mathrm{~kg}^{-h a^{-1}}$ ) nos anos de 2016 (A) e 2017 (B)

Apesar da eficiência da modelagem (EF) ter sido menor que a eficiência dos valores medidos em relação a média, foi constatado pelo coeficiente de determinação (CD) que o modelo explica adequadamente a variação dos dados medidos, exibido pelo CD maior que 1 (Tabela 3).

0 viés da simulação foi determinado pelo cálculo do erro relativo (E), sendo constado que o viés da simulação é menor que o viés das medições $\left(\mathrm{E}<\mathrm{E}_{(95 \%)}\right)$, com intervalo de confiança de 95\%. Pelo teste de $\mathrm{T}$ foi possível constatar que o viés da simulação não foi significativo em relação aos valores medidos (Tabela 3)

Através da análise de LOFIT observou-se que o modelo computacional Century foi eficiente em simular o estoque de carbono orgânico do solo. Vários autores já relataram que o modelo pode ser utilizado com confiabilidade para tal finalidade (Leite et al., 2004; Oliveira et al., 2014; Althoff et al., 2016, 2018). 
Tabela 3. Adequação entre os valores medidos e simulados do estoque de carbono do solo de referência (SR) e dos tratamentos com doses de adubação nitrogenada (0, 30, 70 e 95 kg.ha-1).

\begin{tabular}{|l|c|c|c|c|c|}
\hline & \multirow{2}{*}{ SR } & \multicolumn{4}{|c|}{ DOSES DE N (kg.ha-1) } \\
\cline { 3 - 6 } & & $\mathbf{0}$ & $\mathbf{3 0}$ & $\mathbf{7 0}$ & $\mathbf{9 5}$ \\
\hline LOFIT & $4,26 \times 10^{7}$ & $1,52 \times 10^{8}$ & $2,20 \times 10^{8}$ & $2,70 \times 10^{8}$ & $1,34 \times 10^{8}$ \\
\hline MSLOFIT & $1,71 \times 10^{8}$ & $2,28 \times 10^{9}$ & $3,30 \times 10^{9}$ & $3,51 \times 10^{9}$ & $2,26 \times 10^{9}$ \\
\hline MSE & $1,03 \times 10^{8}$ & $1,06 \times 10^{9}$ & $2,49 \times 10^{9}$ & $2,30 \times 10^{9}$ & $7,15 \times 10^{8}$ \\
\hline Fc=MSLOFIT/MSE & 1,66 & 2,14 & 1,33 & 1,53 & 3,16 \\
\hline Ft $_{(5 \%)}$ & 6,39 & 6,39 & 6,39 & 6,39 & 6,39 \\
\hline RMSE $_{\text {RMSE }}(95 \%)$ & 5,82 & 9,45 & 13,85 & 14,24 & 8,32 \\
\hline $\mathrm{EF}_{\mathrm{CD}}$ & 6,96 & 4,04 & 6,45 & 6,58 & 3,47 \\
\hline $\mathrm{E}$ & $-0,42$ & $-0,30$ & $-0,13$ & $-0,40$ & $-0,46$ \\
\hline $\mathrm{E}_{(95 \%)}$ & 2,41 & 3,32 & 18,41 & 3,83 & 2,24 \\
\hline $\mathrm{M}$ & 2,92 & 3,79 & 1,18 & 4,71 & 4,10 \\
\hline $\mathrm{T}$ & 15,59 & 17,72 & 28,59 & 27,59 & 14,79 \\
\hline $\mathrm{T}_{(2,5 \%)}$ & 2920,42 & 1999,61 & 1280,36 & 2962,70 & 2099,99 \\
\hline
\end{tabular}

LOFIT: Falta de ajuste; MSLOFIT: Quadrado médio da falta de ajuste; MSE: Quadrado médio do erro; Fc: F calculado; Ft: F tabelado; RMSE: Raiz do quadrado médio do erro; S: Desvio padrão; EF: eficiência do modelo; CD: Coeficiente de determinação; E: Erro relativo; M: Diferença média; t: T calculado; $\mathrm{T}_{(2,5 \%)}$ : $\mathrm{T}$ tabelado

O modelo não foi eficiente em simular os valores do estoque de nitrogênio do solo, por apresentar diferença significativa entre os valores simulados com os medidos pelo teste de $\mathrm{F}(\mathrm{P} \leq 0,05)$. A inadequação do modelo também foi constatada pelos procedimentos estatísticos RMSE, EF, CD e E (Tabela 4).

Tabela 4. Adequação entre os valores medidos e simulados do estoque de nitrogênio do solo de referência (SR) e dos tratamentos com doses de adubação nitrogenada (0, 30, 70 e 95 kg.ha-1)

\begin{tabular}{|l|c|c|c|c|c|}
\hline \multirow{2}{*}{} & \multirow{2}{*}{ SR } & \multicolumn{4}{|c|}{ DOSES DE N (kg.ha-1) } \\
\cline { 3 - 6 } & & $\mathbf{0}$ & $\mathbf{3 0}$ & $\mathbf{7 0}$ & $\mathbf{9 5}$ \\
\hline LOFIT & $2,04 \times 10^{6}$ & $1,30 \times 10^{7}$ & $1,74 \times 10^{7}$ & $1,13 \times 10^{7}$ & $1,38 \times 10^{7}$ \\
\hline MSLOFIT & $8,15 \times 10^{6}$ & $1,17 \times 10^{8}$ & $1,57 \times 10^{8}$ & $9,06 \times 10^{7}$ & $1,24 \times 10^{8}$ \\
\hline MSE & $7,45 \times 10^{5}$ & $1,62 \times 10^{6}$ & $1,00 \times 10^{6}$ & $7,44 \times 10^{5}$ & $1,55 \times 10^{6}$ \\
\hline Fc=MSLOFIT/MSE & 10,94 & 72,64 & 156,44 & 121,73 & 80,26 \\
\hline Ft $_{(5 \%)}$ & 7,71 & 4,41 & 4,41 & 4,49 & 4,45 \\
\hline RMSE $_{\text {RMSE}}(95 \%)$ & 15,71 & 27,38 & 29,39 & 26,06 & 27,48 \\
\hline EF & 12,92 & 7,01 & 5,28 & 5,40 & 6,72 \\
\hline CD & $-2,74$ & $-8,06$ & $-17,37$ & $-15,22$ & $-8,93$ \\
\hline E & 0,37 & 0,12 & 0,06 & 0,07 & 0,11 \\
\hline $\mathrm{E}_{(95 \%)}$ & $-14,27$ & 25,23 & 28,24 & 24,95 & 25,49 \\
\hline M & 29,10 & 22,34 & 16,78 & 16,26 & 21,40 \\
\hline T & $-638,38$ & 1141,84 & 1320,55 & 1121,49 & 1175,53 \\
\hline Ttab & $-0,74$ & 0,90 & 1,33 & 1,30 & 0,94 \\
\hline
\end{tabular}

LOFIT: Falta de ajuste; MSLOFIT: Quadrado médio da falta de ajuste; MSE: Quadrado médio do erro; Fc: F calculado; Ft: F tabelado; RMSE: Raiz do quadrado médio do erro; S: Desvio padrão; EF: eficiência do modelo; CD: Coeficiente de determinação; E: Erro relativo; M: Diferença média; t: T calculado; $\mathrm{T}_{(2,5 \%)}$ : T tabelado. 


\section{Conclusões}

A remoção da floresta e implantação da agricultura causa redução do estoque de carbono e nitrogênio do solo, sendo o compartimento lento o que tem maior perda absoluta.

Aplicação de N na dose de $95 \mathrm{~kg}$ ha $^{-1}$ no cultivo do milho em plantio direto favorece a manutenção do estoque de carbono do solo, pelo maior acúmulo de biomassa.

Para as condições climáticas da Microrregião do Brejo Paraibano o modelo computacional Century 4.0 é adequado para realizar as simulações do estoque de carbono do solo, já para o estoque de nitrogênio o modelo não é indicado.

\section{Conflito de interesses}

Os autores declaram não haver conflito de interesses.

\section{Referências}

AESA - Agência Executiva de Gestão das Águas. Climatologia. 2018. Disponível em: <http://www.aesa.pb.gov.br/aesa-website/meteorologia-chuvas/climatologia/>. Acesso em: 12 mar. 2018.

Althoff, T. D.; Menezes, R. S. C.; Carvalho, A. L.; Pinto, A. S.; Santiago, G. A. C. F.; Ometto, J. P. H. B.; Randow, C.; Sampaio, E. V. S. B. Climate change impacts on the sustainability of the firewood harvest and vegetation and soil carbon stocks in a tropical dry forest in Santa Teresinha Municipality, Northeast Brazil. Forest Ecology and Management, v. 360, p. 367-375, 2016. https://doi.org/10.1016/j.foreco.2015.10.001

Althoff, T. D.; Menezes, R. S. C.; Pinto, A. S.; Pareyn, F. G. C.; Carvalho, A. L.; Martins, J. C. R.; Carvalho, E. X.; Silva, A. S. A.; Dutra, E. D.; Sampaio, E. V. S. B. Adaptation of the Century Model to simulate $\mathrm{C}$ and $\mathrm{N}$ dynamics of Caatinga dry forest before and after deforestation.

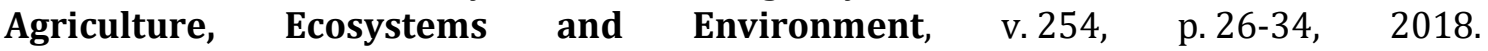
https://doi.org/10.1016/j.agee.2017.11.016

Barreto, A. C.; Freire, M. B. G. S.; Nacif, P. G. S.; Araújo, Q. R.; Freire, F. J.; Inácio, E. S. B. Fracionamento químico e físico do carbono orgânico total em um solo de mata submetido a diferentes usos. Revista Brasileira de Ciência do Solo, v. 32, n. 4, p. 1471-1478, 2008. https://doi.org/10.1590/S0100-06832008000400011

Bayer, C.; Gomes, J.; Zanatta, J. A.; Vieira, F. C. B.; Piccolo, M. C.; Dieckow, J.; Six, J. Soil nitrous oxide emissions as affected by long-term tillage, cropping systems and nitrogen fertilization in Southern Brazil. Soil and Tillage Research, v. 146, n. B, p. 213-222, 2015. https://doi.org/10.1016/j.still.2014.10.011

Bortolon, E. S. O.; Mielniczuk, J.; Tornquist, C. G.; Lopes, F.; Fernandes, F. F. Simulação da dinâmica do carbono e nitrogênio em um Argissolo do Rio Grande do Sul usando modelo Century. Revista Brasileira de Ciência do Solo, v. 33, n. 6, p.1635-1646, 2009. https://doi.org/10.1590/S0100-06832009000600012

Costa, F. S.; Bayer, C.; Zanatta, J. A.; Mielniczuk, J. Estoque de carbono orgânico no solo e emissões de dióxido de carbono influenciadas por sistemas de manejo no sul do Brasil. Revista Brasileira de Ciências do Solo, v. 32, p. 323-332, 2008. https://doi.org/10.1590/S0100-06832008000100030 
Dentener, F.; Drevet, J.; Lamarque, J. F.; Bey, I.; Eickhout, B.; Fiore, A. M.; Hauglustaine, D.; Horowitz, L. W.; Krol, M.; Kulshrestha, U. C.; Lawrence, M.; Galy-Lacaux, C.; Rast, S.; Shindell, D.; Stevenson, D.; Van Noije, T.; Atherton, C.; Bell, N.; Bergman, D.; Butler, T.; Cofala, J.; Collins, B.; Doherty, R.; Ellingsen, K.; Galloway, J.; Gauss, M.; Montanaro, V.; Müller, J. F.; Pitari, G.; Rodriguez, J.; Sanderson, M.; Solmon, F.; Strahan, S.; Schultz, M.; Sudo, K.; Szopa, S.; Wild, O. Nitrogen and sulfur deposition on regional and global scales: A multimodel evaluation. Global Biogeochemical Cycles, v. 20, n. 4, p. 1-21 2006. https://doi.org/10.1029/2005GB002672

Kayler, Z.; Janowiak, M.; Swanston, C. Global carbon. Alban: Climate Change Resource Center, 2017. Disponível em: <https://www.fs.usda.gov/ccrc/topics/global-carbon>. Acesso em: 27 ago. 2019.

Leite, L. F. C.; Mendonça, E. S.; Machado, P. L. O. A. Simulação pelo Modelo Century da dinâmica da matéria orgânica de um Argissolo sob adubação mineral e orgânica. Revista Brasileira de Ciência do Solo, v. 28, n. 2, p. 347-358, 2004. https://doi.org/10.1590/ S0100-06832004000200013

Miranda, C. C.; Canellas, L. P.; Nascimento, M. T. Caracterização da matéria orgânica do solo em fragmentos de mata atlântica e em plantios abandonados de eucalipto. Revista Brasileira de Ciência do Solo, v. 31, n. 5, p. 905-916, 2007. https://doi.org/10.1590/ S0100-06832007000500008

Oliveira, J. G.; Gravina, G. A.; Sousa, E. F. Estoque de carbono do solo em função das mudançaso climáticas simuladas com o Modelo Century. Coffee Science, v. 9, n. 1, p. 1-9, 2014. https://doi.org/10.25186/cs.v9i1.509

Ontl, T. A.; Schulte, L. A. Soil carbon storage. Nature Education Knowledge, v. 3, n. 10, 35, 2012.

Parton, B.; Ojima, D.; Del Grosso, S.; Keough, C. CENTURY tutorial: Supplement to CENTURY user's manual. Fort Collins: Colorado State University, NREL Publication, 2001.

Reed, S. C.; Cleveland, C. C.; Townsend, A. R. Functional ecology of free-living nitrogen fixation: A contemporary perspective. Annual Review of Ecology, Evolution and Systematics, v. 42, p. 489-512, 2011. https://doi.org/10.1146/annurev-ecolsys-102710145034

Santos, H. G.; Jacomine, P. K. T.; Anjos, L. H. C.; Oliveira, V. A.; Lumbreras, J. F.; Coelho, M. R.; Almeida, J. A.; Araújo Filho, J. C.; Oliveira, J. B.; Cunha, T. J. F. Sistema Brasileiro de Classificação de Solos. 5. ed. rev. ampl. Brasília: EMBRAPA, 2018.

Scharlemann, J. P. W.; Tanner, E. V.; Hiederer, R.; Kapos, V. Global soil carbon: understanding and managing the largest terrestrial carbon pool. Carbon Management, $v$, 5, n. 1, p. 81-91, 2014. https://doi.org/10.4155/cmt.13.77

Smith, P.; Smith, J. U.; Powlson, D. S.; McGill, W. B.; Arah, J. R. M.; Chertov, O. G.; Coleman, K.; Franko, U.; Frolking, S.; Jenkinson, D. S.; Jensen, L. S.; Kelly, R. H.; Klein-Gunnewiek, H.; Komarov, A. S.; Li, C.; Molina, J. A. E.; Mueller, T.; Parton, W. J.; Thornley, J. H. M.; Whitmore, A. P. A comparison of the performance of nine soil organic matter models using datasets from seven long-term experiments. Geoderma, v. 81, n. 1/2, p. 153-225, 1997. https://doi.org/10.1016/S0016-7061(97)00087-6

Teixeira, P. C.; Donagemma, G. K.; Fontana, A.; Teixeira, W. G. (Eds.). Manual de métodos de análise de solo. 3. ed. Brasília: EMBRAPA, 2017. 
Tedesco, M. J.; Gianello, C.; Bissani, C. A.; Bohnen, H.; Volkweiss, S. J. Análise de solo, plantas e outros materiais. 2. ed. Porto Alegre: Universidade Federal do Rio Grande do Sul, 1995. (Boletim Técnico de Solos, 5).

Tornquist, C. G.; Mielniczuk, J.; Cerri, C. E. Modeling soil organic carbon dynamics in Oxisols of Ibirubá (Brazil) with the Century Model. Soil and Tillage Research, v. 105, n. 1, p. 3343, 2009. https://doi.org/10.1016/j.still.2009.05.005

Zech W.; Senesi, N.; Guggenberger, G.; Kaiser, K.; Lehmann, J.; Miano, T. M.; Miltner, A.; Schrotha, G. Factors controlling humification and mineralization of soil organic matter in the tropics. Geoderma, v. 79, n. 1, p. 117-161, 1997. https://doi.org/10.1016/S00167061(97)00040-2 